\title{
Correlation between Structures and Magnetism in Iron: Ferromagnetism and Antiferromagnetism
}

\author{
Dongkook Lee and Sooncheol Hong* \\ Department of Physics, University of Ulsan, Ulsan 680-749, Korea
}

(Received 11 June 2007)

\begin{abstract}
Even a pure bulk Fe has a complicated magnetic phase and its magnetism is still needed to be clarified. In this study we investigated the magnetism of bcc and fcc bulk Fe with total energy calculations as functions of atomic volume. The full-potential linearized augmented plane wave method was adopted within a generalized gradient approximation. The ground state of bulk Fe is confirmed to be of ferromagnetic (FM) bcc. For fcc structured Fe an antiferromagnetic (AFM) state is more stable compared to FM states which exist as low spin and high spin states. The stable AFM states were found to accompany a tetragonal distortion, while the FM states remained in a cubic symmetry. At an expanded lattice constant a high spin FM state was calculated to be able to be stabilized with significant enhanced magnetic moment compared to the value of the ground state, bcc FM.
\end{abstract}

Keywords : electronic structure, magnetism, iron, density functional theory

\section{Introduction}

Magnetism of a material is quite sensitive to its environment such as reduced dimensionality, reduced coordinate number, and crystal structure. For examples, $\mathrm{MnPt}_{3}$ shows a more stable antiferromagnetic (AFM) state compared to a ferromagnetic (FM) state at a surface even though its bulk magnetic ground state is $\mathrm{FM}$ [1], whereas $\mathrm{MnAu}_{3}$ does a reverse behavior, stabilized in a FM state at a surface in spite of its ground state of AFM in the bulk [2]. More recently, the magnetism of a $\mathrm{Pd}$ thin film was reported to be dependent of its thickness and exhibit an oscillatory behavior. Its ferromagnetism is realized with particular thicknesses and induced by quantum well states [3].

The magnetic ground state of bulk Fe is well known to be of bcc FM, but metastable fcc and hcp Fe can also be synthesized thanks to recently developed state-of-the-art technology [4-6]. Even though the metastable fcc Fe has been studied for several decades [7-10] because of both fundamental significance and practical application concerns, its magnetism is still controversial. A pioneering study on fcc Fe was done by Moruzzi et al. [11] who

*Corresponding author: Tel: +82-52-259-2331,

Fax: +82-52-259-1693, e-mail: schong@mail.ulsan.ac.kr found that fcc Fe can exist in several magnetic structures and a nonmagnetic (NM) state is more stable compared to FM states, even though they did not take account of AFM state. When local density approximation was used as an approximation for exchange-correlation potential, a calculation failed to predict a correct ground state of bcc FM Fe. [10] The more recent developed generalized gradient approximation (GGA) [12, 13] gave the results of the correct ground state of bcc FM for bulk Fe, being used with the all electron full-potential linearized augmented plane wave (FLAPW) method. However, a pseudopotential method and a linear muffin-tin orbital method still resulted in wrong ground states or controversial results [14, 15]. A pseudopotential calculation employing linear augmented plane wave basis was quite consistent with a result of FLAPW method, even though they considered no AFM state [16].

In this study with the FLAPW method [17] within GGA [18], bcc FM, fcc NM, fct AFM, and fcc FM states were taken account of. We confirmed a bcc FM state to be ground for a bulk Fe. For a fcc bulk Fe an AFM state is more stable compared to the other magnetic states of NM and FM states which exist as low and high spin states. The AFM states were found to accompany a tetragonal distortion, while the FM states remained in a cubic symmetry. The notable finding in this study is that 


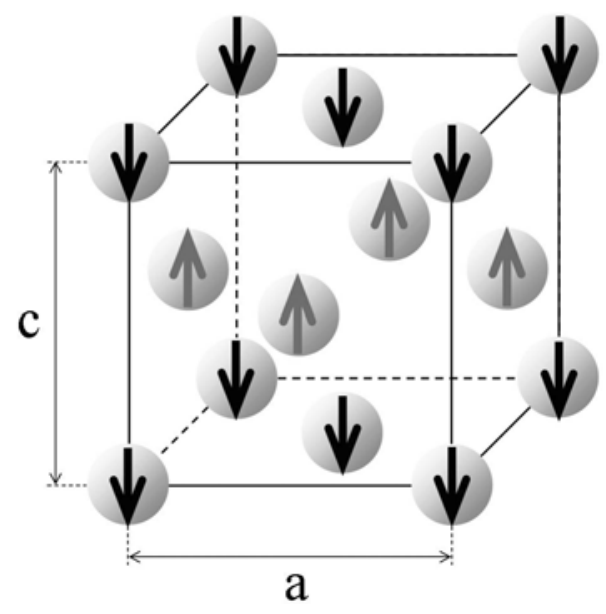

Fig. 1. A schematic view of the unit cell used for fcc AFM Fe.

an FM state with an enhanced magnetic moment can be achieved at an expanded lattice constant.

\section{Methodology}

For bec structured Fe we took account of only FM state and for fcc structured Fe we did AFM state as well as FM one. The AFM coupling between the nearest neighbor $\mathrm{Fe}$ atoms is assumed along z-direction as shown in Fig. 1.

The Schroedinger-like Kohn-Sham equation [19] was solved self-consistently with the FLAPW method. In this method no shape approximation to charge density and potential is adopted and GGA is employed for the exchange-correlation potential. Lattice harmonics, with $l \leq$ 8 , were used to expand the charge density, potential, and wavefunctions inside the muffin-tin (MT) radius of 2.2 a.u. for the Fe atom. An energy cut-off of $13 \mathrm{Ry}$ and a 256 Ry star function cut-off were used for the basis set and for depicting the charge density and potential in the interstitial region, repectively. Integrations over the $3 \mathrm{D}$ Brillouin zone (BZ) were replaced by a summation over $4000 k$-points inside the 3D BZ. Convergence test for numbers of basis functions and $k$-points has been done to check the reliability of our calculation. All core electrons were treated fully relativistically, while valence states were treated scalar relativistically, i.e., considering all the relativistic terms without spin-orbit coupling [20]. Selfconsistency was assumed when the difference between input and output charge (spin) densities was less than 0.5 $\times 10^{-4}$ electrons/a.u. ${ }^{3}$

\section{Calculational Results}

Fig. 2 shows calculated total energies (lower panel) and magnetic moments (upper panel) as functions of atomic

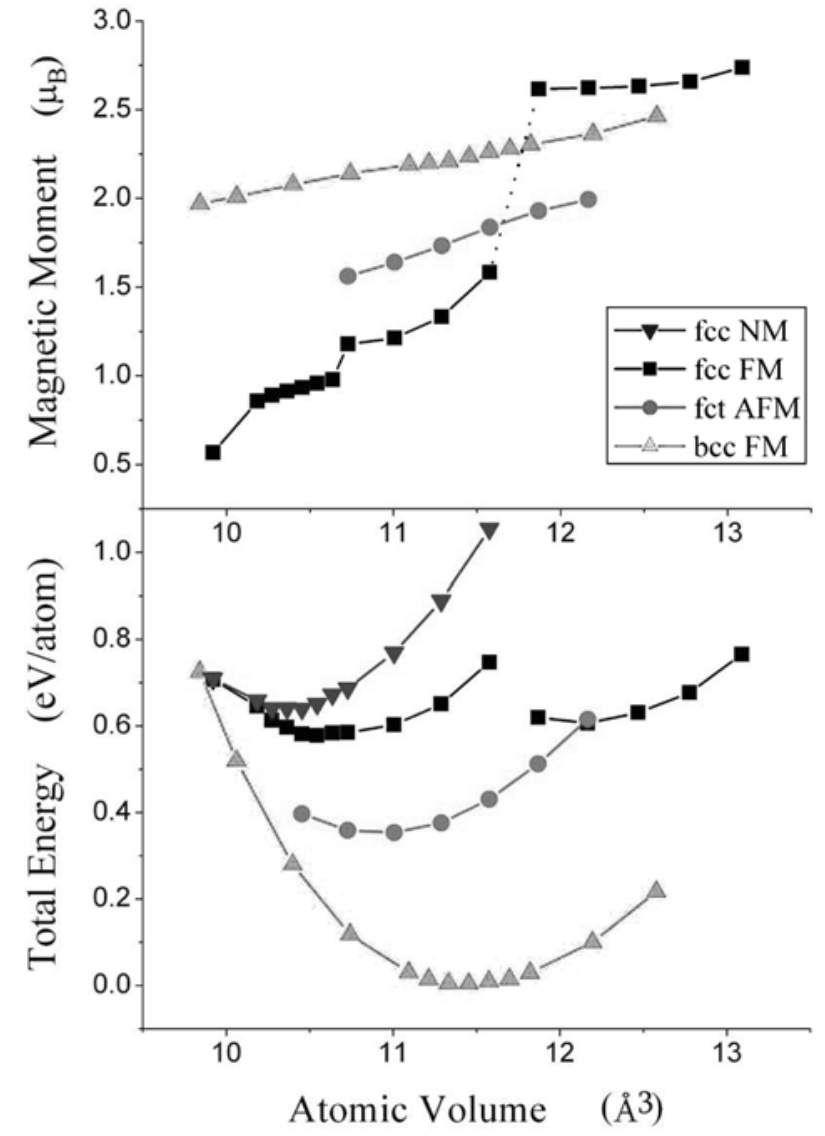

Fig. 2. Magnetic moments and total energies of Fe. Triangles indicate bcc FM; reversed triangles do fcc NM; circles do fct AFM; squares do fcc FM. There are two fcc FM states, LS and HS states.

volume. For fcc structured Fe we also calculated the total energies as functions of $c / a$ ratio. Cubic symmetry is stable for the FM states, but tetragonal distortion takes place for the AFM state. The $c / a$ ratio of the AFM state was calculated to be 1.08 at the equilibrium and the ratio increases linearly as the atomic volume does. The values shown in Fig. 2 are optimized ones for the $c / a$ ratio.

Triangles, diamonds, circles and squares represent bcc FM, fcc NM, fct AFM, and fcc FM states, respectively. As shown in Fig. 2, the bcc FM state was calculated to be most stable and its magnetic moment is $2.24 \mu_{\mathrm{B}}$, which is consistent with experimental findings and confirms reliability of the present calculational method. The fcc FM states are split into two spin states around atomic volume of $11.7 \AA^{3}$ (the corresponding lattice constant $a$ is about 3.6 $\AA$ ); a low spin (LS) state and a high spin (HS) state which are stable for smaller and larger atomic volume ranges, respectively. It is notable that fct AFM is most stable among the magnetic states of the fcc-base structured Fe. This result is distinguishable from a previ- 
ous one by linear-combination-of-atomic-orbital, where an AFM state is almost the same total energy as that of a NM one [21]. The calculated lattice constants at their equilibriums are $2.84,3.53,3.48$, and $3.65 \AA$ for bcc FM, fct AFM, fcc LS FM, and fcc HS FM states, respectively. It may be interpreted as that the AFM coupling expands the bond length between magnetic ions compared to the FM one. The atomic volume of fct AFM Fe at equilibrium is a little smaller than that of bcc FM Fe by about $4.3 \%$.

Since the total energy difference between bcc FM Fe and fct AFM Fe at their equilibriums is about $90 \mathrm{meV}$, the bcc structured FM Fe is proved to be quite stable compared to the other magnetic and crystal structure. As shown in Fig. 2, the total energy differences between the different magnetic states of the fcc (including fct) structured $\mathrm{Fe}$ are not quite significant. In particular the total energy difference (less than $10 \mathrm{meV}$ ) between the LS and HS states in the fcc structured Fe is negligible. Furthermore, the HS FM state is most stable among the present considered states of fcc (including fct) structured Fe at larger lattice constants than that $(3.65 \AA)$ of the HS FM state at its equilibrium. It implies that we may do engineering the magnetic states of an fcc structured Fe, controlling of the lattice constant of an fcc structured Fe by selection of a proper fcc structured substrate.

As shown in upper panel of Fig. 2, the magnetic moments of Fe in bcc FM and fct AFM states increase relatively linearly with increase of atomic volume, whereas the magnetic moment of Fe in the fcc FM state behaves quite complicatedly. The fcc FM states can be classified as LS and HS states basically. For the fcc LS states further classification is possible; there are at least two more abrupt changes in magnetic moment. The unsmooth behavior is also exhibited in the total energies of the fcc LS state. We checked carefully the calculational procedure with the convergence test for numbers of $k$ points and basis functions and self-consistency to confirm the preciseness of our calculation, but no meaningful changes were observed. It is plausible for the unsmooth behaviors to be related to invar property observed in some
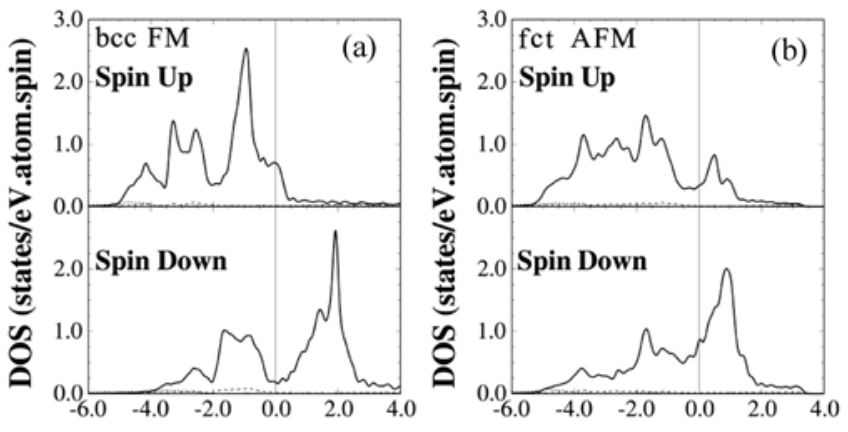

$\mathrm{E}(\mathrm{eV})$
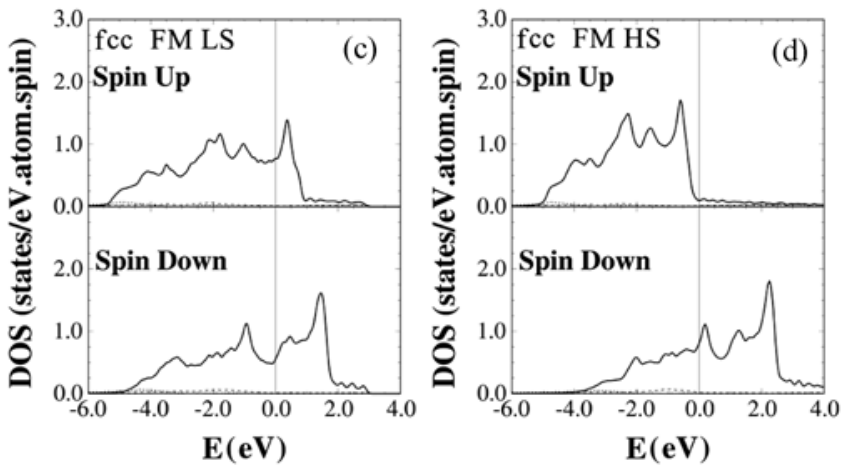

Fig. 3. The spin-polarized layer-projected density of states in (a) bcc FM, (b) fct AFM, (c) fcc LS FM, and (d) HS FM states. The Fermi levels are set to zero.

fcc structured Fe's. Further investigation is needed to clarify the origin of the strange behaviors of total energy and magnetic moment in the fcc LS state.

Spin and charge densities are fundamental physical quantities in density functional theory. Table 1 presents the number of electrons and the magnetic moment within each of muffin-tin spheres for different magnetic states of the fcc and bcc structured Fe. The calculated magnetic moments of bcc FM, fct AFM, fcc LS FM, and fcc HS FM are 2.24, 1.63, 1.04, and $2.64 \mu_{\mathrm{B}}$ at their equilibrium atomic volumes, respectively. The magnetic moment of the fcc HS state is larger by more than $18 \%$, being compared to that of the ground state of the bcc FM. It is noteworthy that an fcc structured Fe can have a large magnetic moment as aforementioned.

Table 1. Numbers of the atom-projected $l$-decomposed spin-up and -down electrons and magnetic moments (in units of $\mu_{\mathrm{B}}$ ) inside each of the muffin-tin spheres.

\begin{tabular}{cccccc}
\hline \hline States & $\begin{array}{c}s \\
(\downarrow)\end{array}$ & $\uparrow \begin{array}{c}p \\
(\downarrow)\end{array}$ & $\uparrow^{d}(\downarrow)$ & Total & $M$ \\
\hline bcc FM & $0.168(0.174)$ & $0.152(0.187)$ & $4.125(1.847)$ & $4.459(2.219)$ & 2.240 \\
fct AFM & $0.189(0.171)$ & $0.198(0.173)$ & $3.781(2.192)$ & $4.181(2.551)$ & 1.630 \\
fcc LS FM & $0.184(0.186)$ & $0.187(0.199)$ & $3.532(2.480)$ & $3.922(2.880)$ & 1.042 \\
fcc HS FM & $0.162(0.170)$ & $0.139(0.172)$ & $4.305(1.631)$ & $4.618(1.981)$ & 2.637 \\
\hline
\end{tabular}


Fig. 3 shows atom(integration over each MT sphere)and $l$-projected density of states (DOS) of (a) bcc FM Fe, (b) fct AFM Fe, (c) fcc LS FM Fe, and (d) fcc HS FM Fe at their own equilibrium atomic volumes. Solid lines represent $d$ states and dotted and dashed lines do $s$ and $p$ states, respectively. Spin up and down states are presented in separate panels. Roughly speaking, the fcc structures have similar DOSs except band narrowing or band broadening depending on their atomic volumes. Being compared to the fcc FM HS state, the fcc FM LS state has a broader band and smaller exchange splitting. The fcc LS Fe has high values of DOS at its Fermi level for both spin up and down states, whereas the DOSs at their Fermi levels of the other Fe's are low for either spin up state or spin down one. Almost completely occupation of the spin up states of the fcc HS Fe is needed to be noted. More detailed discussion on the relationship of observed magnetism to corresponding electronic structure will be given elsewhere.

\section{Summary}

In this study, we investigated the magnetism of bcc and fcc structured bulk Fe using the FLAPW method within GGA by calculating their total energies in NM, FM and AFM states. We confirmed FM bcc to be the ground state of a bulk Fe. For fcc structured Fe an AFM state is most stable compared to NM and FM states which exist as LS and HS states. The fcc AFM Fe were found to be stabilized in a tetragonal distortion, while the FM states remained in a cubic symmetry. The calculated magnetic moments of bcc FM, fct AFM, fcc LS FM, and fcc HS FM are $2.24,1.63,1.04$, and $2.64 \mu_{\mathrm{B}}$ at their equilibrium atomic volumes, respectively. It is found that an fcc HS FM state is achievable with high magnetic moment if a proper substrate is chosen.

\section{Acknowledgment}

This work was supported by the Korean Science and Engineering Foundation grant (No. R01-2007-000-11593-0).

\section{References}

[1] W. Kim, S. C. Hong, J. Seo, S.-J. Oh, H. G. Min, and J.S. Kim, Phys. Rev. B 70, 174453 (2004).

[2] W. Kim, S.-J. Oh, J. Seo, H. G. Min, S. C. Hong, and J.S. Kim, Phys. Rev. B 65, 205407 (2002).

[3] S. C. Hong, J. I. Lee, and R. Wu, Phys. Rev. B 75, 172402 (2007).

[4] D. Qian, X. F. Jin, J. Barthel, M. Klaua, and J. Kirschner, Phys. Rev. B 66, 172406 (2002).

[5] N. Kamakura, A. Kimura, T. Saitoh, O. Rader, K.-S. An, and A. Kakizaki, Phys. Rev. B 73, 094437 (2006).

[6] V. Iota, J.-H. P. Klepeis, C. S. Yoo, J. Lang, D. Haskel, and G. Srajer, Appl. Phys. Lett. 90, 042505 (2007).

[7] C. Berghout, Z. Metallkd. 52, 179 (1961).

[8] W. Schwarzacher, W. Allison, R. F. Willis, J. Penfold, R. C. Ward, I. Jacob, and W. F. Egelhoff, Jr., Solid State Commun. 71, 563 (1989).

[9] K. Haneda, Z. X. Zhou, A. H. Morrish, T. Majima, and T. Miyahara, Phys. Rev. B 46, 13832 (1992).

[10] C. S. Wang, B. M. Klein, and H. Krakaure, Phys. Rev. Lett. 54, 1852 (1985).

[11] V. L. Moruzzi, P. M. Marcus, K. Schwarz, and P. Mohn, Phys. Rev. B 34, 1784 (1986).

[12] H. C. Herper, E. Hoffmann, and P. Entel, Phys. Rev. B 60, 3839 (1999).

[13] Y. M. Zhou, S. C. Wu, and D. S. Wang, Solid State Commun. 111, 101 (1999).

[14] T. Asada and K. Terakura, Phys. Rev. B 46, 13599 (1992).

[15] D. Vanderbilt, Phys. Rev. B 41, 7892 (1990).

[16] J.-H. Cho and M. Scheffler, Phys. Rev. B 53, 10685 (1996)

[17] E. Wimmer, H. Krakauer, M. Weinert, and A. J. Freeman, Phys. Rev. B 24, 864 (1981), and references therein.

[18] J. P. Perdew, K. Burke, and M. Ernzerhof, Phys. Rev. Lett. 77, 3865 (1996); ibid. 78, 1396(E) (1997).

[19] W. Kohn and L. J. Sham, Phys. Rev. 140, A1133 (1965).

[20] D. D. Koelling and B. N. Harmon, J. Phys. C 10, 3107 (1977).

[21] T. C. Leung, C. T. Chan, and B. N. Harmon, Phys. Rev. B 44, 2023 (1991). 